Oikos 122: 1565-1572, 2013

doi: $10.1111 / j .1600-0706.2013 .00298 . x$

(C) 2013 The Authors. Oikos (C) 2013 Nordic Society Oikos

Subject Editor: Dustin Marshall. Accepted 10 March 2013

\title{
Emergent neutrality or hidden niches?
}

\author{
György Barabás, Rafael D'Andrea, Rosalyn Rael, Géza Meszéna and Annette Ostling \\ G. Barabás (dysordys@umich.edu), R. D’Andrea, R. Rael and A. Ostling, Dept of Ecology and Evolutionary Biology, Univ. of Michigan, \\ 830 North University, Ann Arbor, MI 48109-1048, USA. - G. Meszéna, Dept of Biological Physics, Eötvös Loránd Univ., \\ Pázmány Péter sétány 1A, HU-1117, Budapest, Hungary.
}

\begin{abstract}
Emergent neutrality is the idea in community ecology that species interactions may drive a system in a direction where some species become so similar that this similarity will be the primary cause for their coexistence instead of niche differentiation. A recent, widely cited model of emergent neutrality is by Scheffer and van Nes, later applied to species abundance distribution patterns by Vergnon et al. We take issue with the ecological interpretation of this model, demonstrating that it in fact presupposes important differences between superficially similar-looking species. We argue that the temptation to interpret the model as one of emergent neutrality stems from the fact that these differences are unmodeled and therefore hidden, obscuring the underlying coexistence mechanisms. We therefore claim that the model is actually one of hidden niches, and present several alternative ways to make its hidden portions more explicit. These alterations to the model also make its proper interpretation as one of hidden niches more transparent. We also polemize with the claim of Vergnon et al. that multimodality in species abundance distributions is support for their emergent neutrality model: we demonstrate that appropriate stochastic versions of classical resource partitioning or even neutral models can lead to such patterns in a robust way. Observation of these patterns is therefore inconclusive as to the underlying mechanisms that generate them.
\end{abstract}

Within community ecology, one relatively recent idea has been the notion of 'emergent neutrality'. In brief, this school of thought addresses the questions whether and how it is possible for interspecific interactions to drive a community towards a state in which relative competitive abilities differ so little that demographic stochasticity and potential immigration from outside sources will dominate community patterns instead of the details of the species interactions themselves. The problem has an ecological and an evolutionary aspect (Bonsall et al. 2004, Holt 2006, Hubbell 2006, Scheffer and van Nes 2006). In this article we consider the ecological side only. More specifically, we will examine a model that was first proposed by Scheffer and van Nes (2006), and then applied to macroecological patterns by Vergnon et al. (2012). This model, coined 'self-organized similarity' by its original authors and later 'emergent neutrality' by Holt (2006), has received widespread attention (Nee and Colegrave 2006, Holt 2006, Hérault 2007).

The emergent neutrality (EN) model was inspired by the observation that in multispecies competitive LotkaVolterra models along a single trait axis, even if only a handful of species survive in the final equilibrium state, this state is preceded by long transients in which species very similar to the eventual survivors slowly crawl towards extinction. While the time it takes for these transients to disappear will depend on model details (such as the distribution of carrying capacities), the fact that such transient clusters of species emerge is a general phenomenon that is expected to hold as long as fitness is a continuous function of trait value. In that case, the more similar two species are, the more similar their fitnesses will be and so exclusion can be arbitrarily slow between them. The idea behind the EN model is that such transient coexistence can be stabilized by potentially very weak forces due to the similarity of the species' fitnesses. The EN model therefore employs species-specific negative density dependence on top of the usual Lotka-Volterra-style competitive interactions, and indeed, this revised model can produce stable coexistence between species arbitrarily close along the trait axis. This was interpreted by Scheffer and van Nes (2006) and Vergnon et al. (2012) as their model unifying niche and neutral perspectives in community ecology: each cluster of species is segregated by the other via classical resource competition, while species within a cluster coexist mainly due to their similarity. Patterns of clustering similar to those that arise as a stable feature in the EN model have been observed in a variety of systems, and in particular Scheffer and van Nes (2006), Segura et al. (2011), Vergnon et al. (2012) and Yan et al. (2012) have suggested that these patterns are evidence in favor of the EN model.

More recently, further empirical support for the EN model has been presented by Vergnon et al. (2012). A new trend in studying species abundance distribution (SAD) 
patterns has been to identify whether SADs, which have long been thought to possess a single mode, are in fact multimodal (McGill et al. 2007, Dornelas and Connolly 2008). While the frequency of multimodal SADs relative to unimodal ones found in nature is unknown, there is some evidence that such patterns might well be common. Vergnon et al. (2012) provided an elegant theoretical underpinning to this phenomenon, by showing that the EN model can produce multimodal SAD patterns in a robust way. Furthermore, they argued that other community assembly theories cannot produce multimodal SADs, and hence their existence should be taken as support for the EN model.

The EN model is certainly a candidate theory of multimodality in SADs, and of community assembly more broadly. However, we are not convinced by Vergnon et al.'s (2012) study that the EN model would produce multimodality more consistently than other community assembly theories, such as resource partitioning or neutral models. Equally important, we dispute the claim of Scheffer and van Nes (2006), Holt (2006), and Vergnon et al. (2012) that the model provides a unification of niche and neutral modes of community assembly. Instead, we argue, it relies on unmodeled species differences to generate coexistence. Therefore, these species are only apparently similar: properly accounting for those unmodeled portions will reveal the important coexistence-generating species differences that were hidden in the original formulation. We propose several alternative ways in which these implicit, hidden differences might be accounted for. Whether any such revisions to the model would still lead to multimodal SADs remains to be seen.

\section{Does neutrality emerge from the theory of emergent neutrality?}

The ecological part of the EN model (Scheffer and van Nes 2006, Vergnon et al. 2012) assumes Lotka-Volterra competition along a unidimensional, circular trait axis, on top of which there is independent density regulation of each phenotype. The dynamics of this model lead to the emergence of clusters of arbitrarily similar species that stably coexist, separated by exclusion zones where no species survive (Fig. 2A'). The interpretation of this coexistence pattern according to Vergnon et al. (2012) is that species within one cluster '...coexist in essentially the same niche, thereby bridging a gap between niche and neutral theory'. This coexistence even between arbitrarily similars is ensured by the extra densitydependent regulation term in their equations (Eq. 1 in the Methods), which acts on each species independently, regardless of how close they might be along the trait axis.

The authors' interpretation of the mode of coexistence in their model is tempting, but it is in fact the very opposite of what is actually going on. We argue below that, in adding regulation that is species-specific, Scheffer and van Nes (2006) and Vergnon et al. (2012) are inadvertently introducing unspecified, hidden species differences into their model. These enable the regulating agents to tell apart species that seem to be arbitrarily similar when measured on the one trait axis that is explicitly modeled.

The mathematical feature of the model obscuring the fact that species which are close on the trait axis possess hidden differences can be seen on Fig. 2A. The figure depicts the EN model's competition kernel (competitive effect of two species on one another as a function of their trait difference). Notice that, on top of the smooth curve, there is an infinitely sharp peak at zero trait difference - this is how the model phenomenologically incorporates the extra density regulation imposed on each species. However, if species indeed only differ in the single trait of the model, then biological realism rules that competitive effect should depend smoothly on trait difference (Adler and Mosquera 2000, Barabás et al. 2013). Consider bird species which differ only in bill depth. It stands to reason that species with ever so similar bill depths should be regulated ever so similarly - if the difference in trait is minuscule then no potential regulating agents would be able to tell them apart in the first place (Meszéna 2005). The EN model does not fulfill this criterion. In fact, the model insists on extra regulating factors that affect each species individually regardless of how closely placed on the trait axis. Therefore, the only way in which the model can remain biologically realistic is by assuming that the modeled trait is not the only thing in which species differ from one another.

Of course, the EN model by itself does not uniquely determine what those species differences are, but that they must be present is clear from our argument. It is easy to think of specific examples. In line with the original interpretation of the extra density regulation term given by Scheffer and van Nes (2006), one could imagine that the community has species-specific predators. In that case, two species that are very close on the modeled trait axis might have, say, different coat patterns, providing camouflage from one (but not the other) predator. If the coat patterns were not different, the predators would not be able to tell the two species apart and so individual regulation of the species would become impossible. Another option would be for members of the community to differ in their blood chemistry, making them protected from (and exposed to) different pathogen species. Then, if the pathogens are all blood type-specific, the maximum number of species that can coexist while occupying the exact same position along the original trait axis will be equal to the number of different blood types. Yet another possibility is to have spatial structure with some environmental gradient - then two species that are very similar along the modeled axis could have different environmental tolerances, and thus live in separate patches and coexist.

The number of hypothetical scenarios is infinite. What they all have in common is the involvement of crucial, coexistence-generating species differences, in the absence of which it is impossible to mediate negative density dependence for each species independently. Conforming to the modern usage of the word, the implicit species differences in the model are therefore niche differences, where the term is not restricted to resource competition (Chesson 1991, 2000, Leibold 1995, Chesson et al. 2001, Amarasekare 2003. Chase and Leibold 2003, Meszéna et al. 2006, Adler et al. 2007, Levine and HilleRisLambers 2009).

Returning to the question of the EN model's interpretation as one of emergent neutrality: the claim by Scheffer and van Nes (2006) and Vergnon et al. (2012) that species close along the modeled trait axis coexist mainly due to their 
similarities does not hold. Since, as we have seen, the coexisting species of the model actually do differ in ecologically important ways even if they happen to be similar along the particular trait axis under scrutiny, the EN model cannot be one of emergent neutrality. The essential ingredients of neutrality are ecological equivalence and pure ecological drift. None of these ingredients are incorporated into the EN model: first, it is a purely deterministic model, precluding the possibility for ecological drift; second, it produces stable (as opposed to neutrally stable) coexistence between species; third, species that are close along the modeled trait axis are actually very different in some other trait(s) not explicitly accounted for.

We therefore propose that is it better to acknowledge this feature of the EN model right from the outset, and to interpret it as a model of hidden niches instead of emergent neutrality. What we mean by 'hidden' niches is that the details of the interactions leading to niche segregation along the unmodeled traits are treated in a purely phenomenological manner. If one lacks sufficient information about, or is simply uninterested in the details of the mechanism bringing about the species-specific density regulation, then the EN model provides a sound and potentially useful nonmechanistic model. As long as we can keep in mind that 'being close on the trait axis' does not translate into 'occupying the same niche' or 'being ecologically similar', there is no reason one could not think about community dynamics in this particular way.

Our proposed interpretation notwithstanding, the model does have two serious limitations. First of all, since the details of the hidden mechanism are obscured, quantitative predictions should be used judiciously. In particular, the claim of Vergnon et al. (2012) that this model produces multimodal SADs as a robust feature might sensitively depend on how exactly the hidden mechanism actually operates. Second, a model relying on hidden differences to explain coexistence does raise questions about its own utility. Consider two species along the modeled trait axis that cannot coexist without the extra density regulation term. Their coexistence is then unexplained by the part of the model that is mathematically explicit, but is explained by the unmodeled mechanism. If that is truly the case, then one should not bother with the mathematical model to begin with, as coexistence is adequately explained by implicit, verbal arguments in the first place.

To ultimately be able to deal with these problems, the hidden parts of the model have to be made mathematically explicit. The most straightforward way of doing this is by incorporating the hidden mechanism via another niche dimension, leading to a multidimensional niche model. An example of this modeling strategy can be seen on Fig. 1. We now have two independent trait axes. In line with our earlier discussion, the extra trait axis could represent a wide array of different things. (What that trait is in a given ecological situation, how species interactions depend on it, whether the trait is discrete or quantitative, etc. are empirical questions. Here we are merely showing how such a strategy may be implemented in principle; the contingent details of the model will vary on a case-to-case basis.) Initially, 200 species were randomly placed in this two-dimensional trait space, then the system was simulated until equilibrium was reached
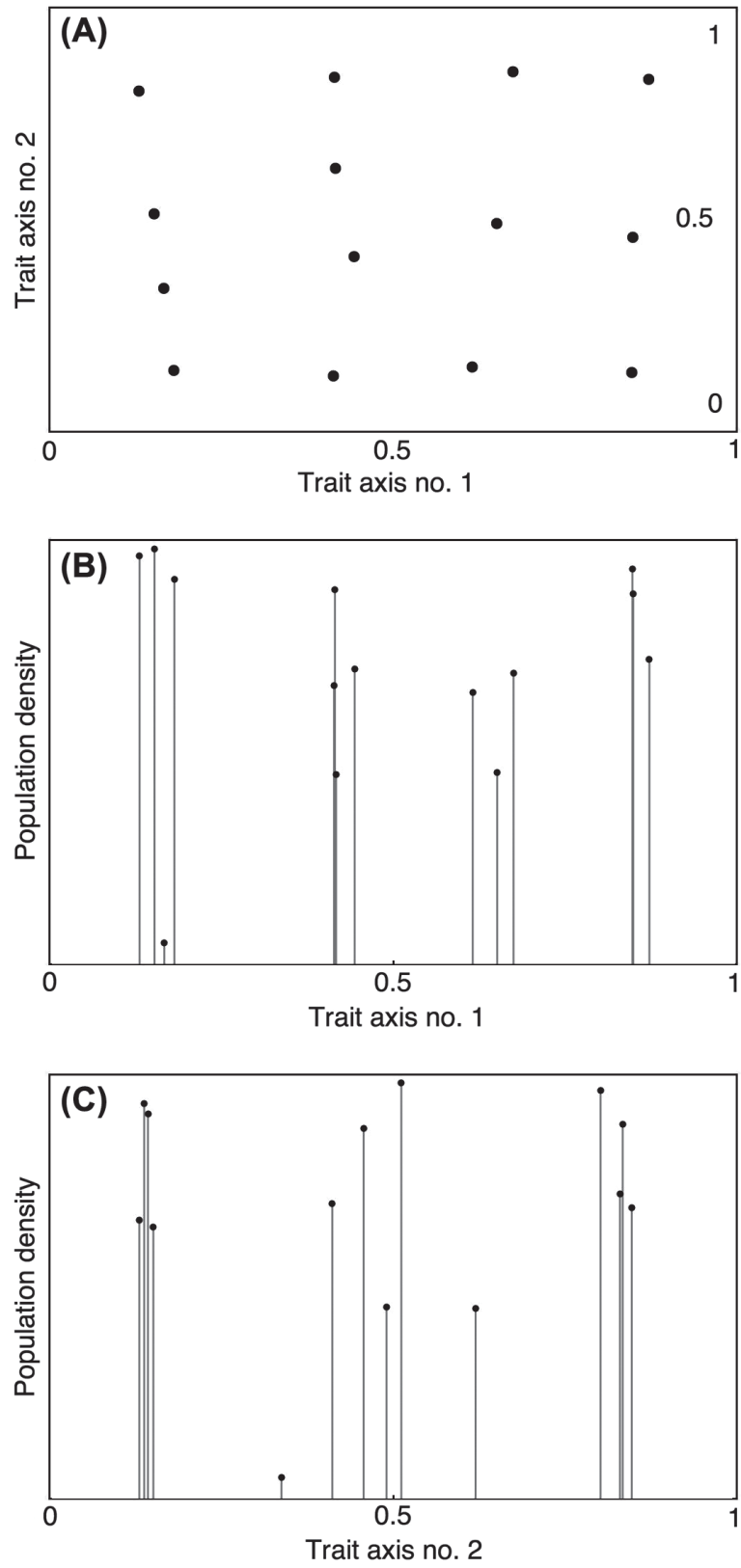

Figure 1. Results of simulated Lotka-Volterra competition with two independent circular trait axes. Initially, 200 species were randomly positioned in this trait space with uniform densities. (A) The distribution of species at equilibrium; the abundances are not shown. Limiting similarity is evident, but only if one considers the trait space as a whole. On (B) and (C) one can see the surviving species projected onto the first and second trait axes, respectively. Notice that separate clusters of species become apparent: without considering both trait axes, one would get the impression that species within these clusters stably coexist without niche differentiation.

(Methods). When looking at the trait positions of survivors within the whole trait space (Fig. 1A) the species are more or less regularly spaced out, but if we only acknowledge the existence of a single trait axis (Fig. 1B-C), we would get the impression that clusters of species stably coexist without niche differentiation - the pattern generated by the original EN model. 
It is also possible to modify the EN model in a slightly different spirit. After all, one could insist that the single trait axis be truly the only thing in which species should differ. For example, resource use could be determined by body size, and this same trait could be the basis for predators to choose their prey. In this case it has to be taken into account that no predator has infinite powers of discrimination: even if they are very apt at telling body sizes apart, there must come a point beyond which two prey items are too similar to be differentiated.

This scenario can be implemented by slightly smearing the infinitely sharp top-down regulation over the trait axis. Figure 2A and 2B depict this difference. Figure 2A corresponds to the competition kernel of the original EN model. In turn, Fig. 2B shows the case where the extra densitydependent regulation, though extremely species-specific, is not infinitely so. Figure $2 \mathrm{~A}^{\prime}$ and $2 \mathrm{~B}^{\prime}$ are corresponding equilibrium coexistence patterns, obtained via simulating a Lotka-Volterra model along a circular trait axis with 200 randomly placed species starting with equal abundances (Methods). Figure 2A' yields what is expected under the EN model: exclusion zones and clusters of species in between, and within the clusters species can be arbitrarily similar. On Fig. 2B', where everything is the same as before except for the competition kernel, we see much the same thing, except that the species within the clusters cannot be arbitrarily similar. The differences within a cluster are governed by the width of the extra regulatory term, i.e. the width of the 'tip' of the kernel on Fig. 2B. Incidentally, the same is true for the EN simulation: since the width of the 'tip' is zero in that case, no limit to the similarity of the species within the clusters is observed. As previously argued, this would only be biologically realistic if there were other, hidden differences between the species, leading back to our previous, multidimensional modeling strategy.

In summary, the EN model is actually a model of hidden niches, where clusters of coexisting species emerge due to differences in the modeled trait, and species within a cluster coexist via differing in some other, unmodeled trait. Since the effects of this extra trait are handled on a purely phenomenological basis, it remains implicit in the model. Due to its hidden nature, it is not immediately apparent that two coexisting species which are very similar along the explicitly modeled trait axis are actually different in the hidden one. If one overlooks this fact, there will be a temptation to interpret the model as one that unifies niche and neutral perspectives of biodiversity maintenance. Reminding oneself that those species are only superficially similar
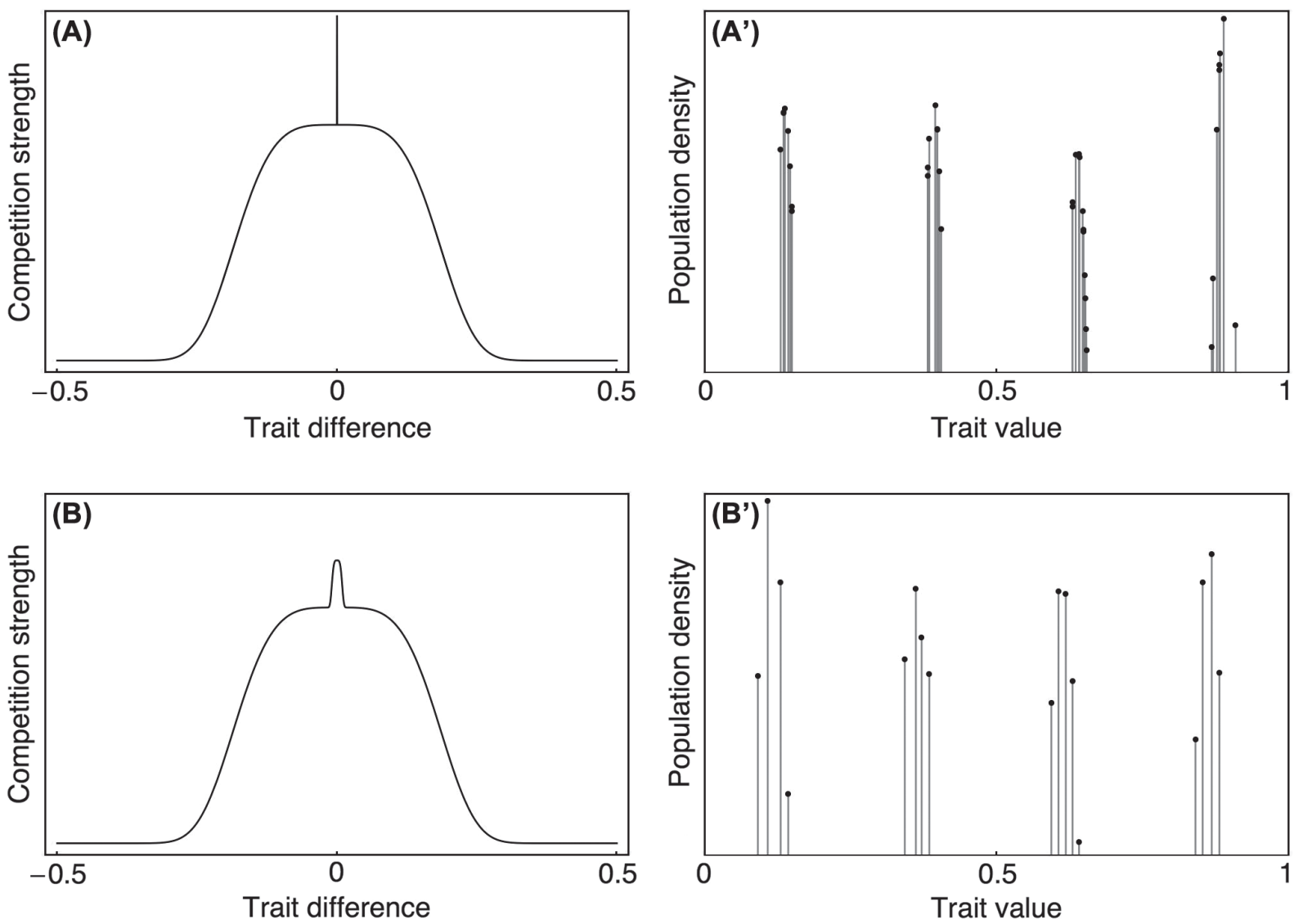

Figure 2. Results of simulated Lotka-Volterra competition using two different competition kernels. In both cases, 200 species were randomly placed along the trait axis with uniform densities. Though the trait positions were randomized, they are the same between the two runs. (A) is a competition kernel with extra self-interaction added, which only influences the species in question and nobody else (this is the approach of the original EN model); (A') is the resulting equilibrium community. (B) is a kernel like the one in (A), except that the infinitely sharp peak has been smeared out slightly. (B') Observe that this kernel leads to a coexistence pattern where separate clumps of species appear, but within each clump the species are separated by a distance that roughly corresponds to the width of the 'tip' of the kernel. 
opens the avenue for interpreting the model's results correctly, as well as for improving on the model by making its hidden portions more explicit.

\section{Multimodality in SADs}

Problems with its interpretation notwithstanding, one potentially important aspect of the EN model is that it offers an explanation to the phenomenon of multimodality in SADs. Vergnon et al. (2012) emphasize that currently existing SAD theories, such as resource partitioning (MacArthur 1960, 1970, Sugihara 1989) or neutral (Hubbell 2001) models, cannot produce such patterns at all. If true, this would not only provide empirical support for the EN model, but would also be an argument that SAD patterns can usefully inform one about underlying processes. As this capacity of SADs is frequently questioned (McGill 2003), the debate over their importance would be seen in a new light.

We argue however that classical resource partitioning and neutral models are in fact able to produce multimodality in SADs, thus contesting the EN model's claim of being the sole contender for explaining the phenomenon. We also ask the question whether an EN model modified as we described in the previous section would still robustly produce multimodal SADs.

The widely held consensus that resource partitioning and neutral models predict strictly unimodal SADs probably stems from the fact that their predicted mean patterns are always unimodal (see Hubbell 2001 and Volkov et al. 2003 for the neutral case). In nature, however, a particular community will not correspond to some theoretical mean, but instead to a single realization of the stochastic process. Individual realizations may well differ from the average. Moreover, such a discrepancy between individual realizations and the underlying mean pattern could be the norm - this will always happen when the mean and the mode of a distribution do not coincide. This implies that in principle a stochastic model could easily produce multimodal patterns even if the underlying mean pattern is unimodal.

The basis for the EN model is Lotka-Volterra competition along a circular trait axis with uniform carrying capacities and possibly immigration (but without the extra density-regulation term). This offers a simple resource partitioning model which does not produce multimodality in the deterministic limit. We examined a stochastic version of this competition model, with immigration from a regional pool, to see if it produced multimodality. In other words, we examined a stochastic version of the EN model with the extra density-dependent regulation term removed. The model is similar in spirit to other 'stochastic niche models' in the literature (Chave et al. 2002, Tilman 2004, Gravel et al. 2006, Haegeman and Loreau 2011) which incorporate both niche differentiation and the stochastic elements of neutral theory, and also incorporates the resource competition that formed the basis of classical resource partitioning based models of the species abundance distribution (MacArthur 1960, Sugihara 1989). The model produces clusters of species as a stable feature similar to the EN model (Fig. 3). However, the reason for them is the stochastic immigration, not extra negative density

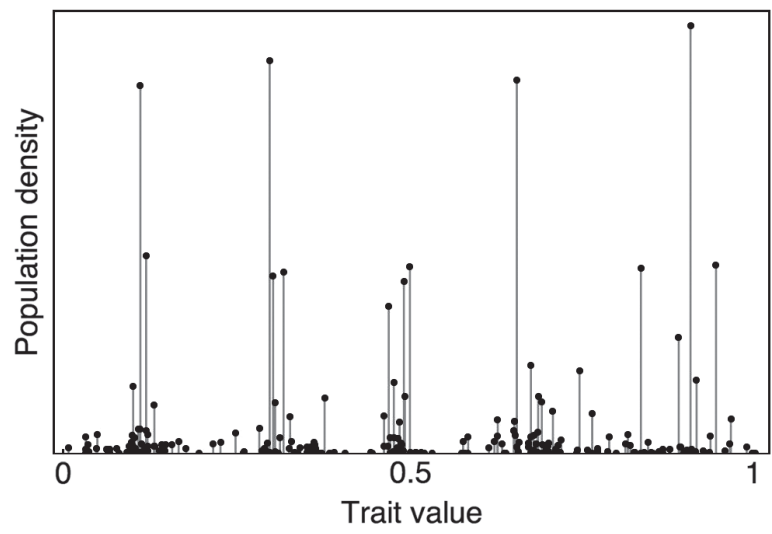

Figure 3. A sample run of the stochastic niche model near stationary equilibrium. Notice the clustering of species around a handful of dominant ones. The subdominant species persist due to the fact that their deterministic decline towards extinction is so slow that stochastic immigration still keeps them at a reasonable abundance.

dependence. Species within a cluster therefore are actually very similar in this model, 'almost neutral', in the sense that drift and immigration play an important role in their relative dynamics. In the original EN model, as each species is independently stabilized, this would not hold.

To examine whether this stochastic niche model would produce multimodality, we performed 100 stochastic simulations until stationary equilibrium was reached, and tested each resulting SAD for multimodality (Methods). This revealed that there is in fact a $50 \%$ chance that any individual realization of the stochastic process ends up multimodal (in stark contrast with the prediction based on the underlying theoretical mean distribution, which predicts 100\% unimodality; Fig. 4A). This estimate should be conservative, as we were only testing for two modes at most, missing out on patterns with three or more modes. Also, due to methodological difficulties in fitting multiple modes which do not occur when fitting a single mode (Dornelas and Connolly 2008), optimal fitting of two modes was more difficult to achieve than of a single mode, biasing our results towards unimodality. In short, our results indicate that multimodal SADs could be expected to arise from individual runs of our stochastic niche model at least half the time.

But are niche interactions even needed for this multimodality to arise? To answer this question, we repeated the simulations described above with all competition coefficients set to be equal to one, i.e. we simulated a neutral community (Methods). For this model the underlying mean SAD pattern can be obtained analytically (Hubbell 2001): it is the so-called zero-sum multinomial distribution. This distribution is unimodal. However, according to our simulation results, individual runs of the neutral model are multimodal $50 \%$ of the time (Fig. $4 \mathrm{~B}$ ). Therefore, the neutral model produces multimodality just as often as the stochastic niche model does.

These results suggest that multimodality detected in nature may well be a signature of demographic stochasticity and not represent any other underlying ecological processes. Vergnon et al. (2012) already acknowledged that multimodal SADs may arise even in the absence of the 


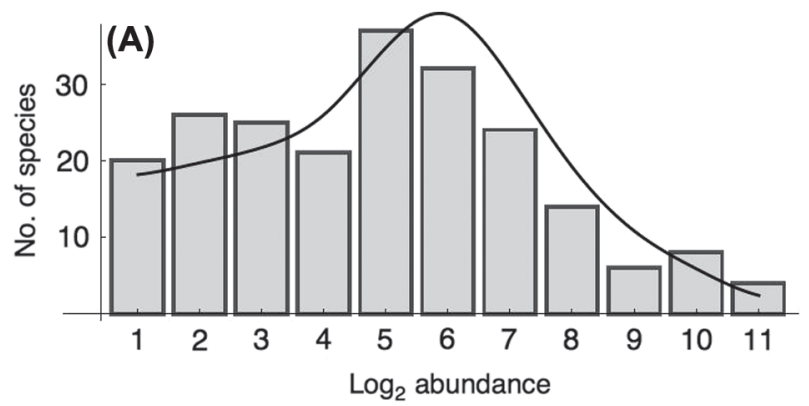

(B)

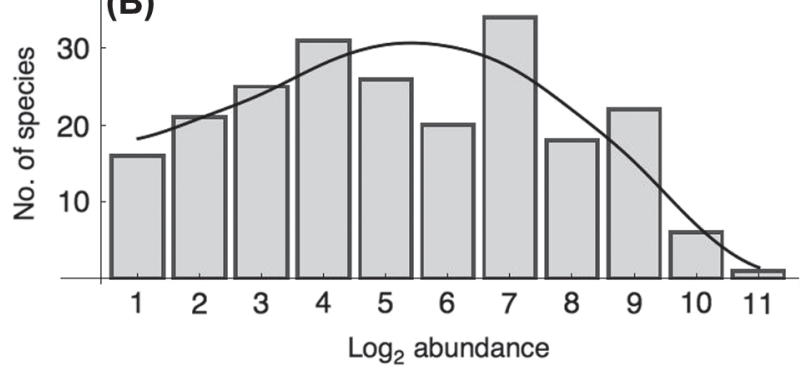

Figure 4. Multimodality in SADs produced by (A) stochastic niche, and (B) neutral dynamics. The bars represent a single realization of the stochastic process that ended up significantly multimodal; the solid lines are the average SADs over 100 simulations. In both the niche and the neutral case, significantly multimodal patterns were produced half the time, while the mean over all runs was unimodal. This demonstrates that judging the ability of a stochastic model to produce a certain kind of pattern based on properties of the underlying theoretical mean can be very misleading.

mechanism proposed by the EN model, e.g. due to environmental heterogeneity, or differences in niche suitability. Here we showed that demographic stochasticity is another potential candidate for explaining multimodality. The sensitivity of this multimodality to parameters like the width of the competition kernel or the strength of immigration is as yet unknown, as we carried out simulations with these each fixed at a particular value. We chose an immigration rate that is thought to be approximately realistic for a known community (in particular Barro Colorado Island; Methods). We chose a competition kernel width that produces roughly the same number of clusters of species on the trait axis as cases of the EN model examined by Vergnon et al. (2012).

Nevertheless, should this result prove to be robust against changes in these parameters, it would also have the unfortunate corollary that multimodality in SADs is not a phenomenon that can convincingly inform one about underlying ecological processes. The debate over the utility of such patterns is therefore not resolved here; we merely have another reason to be somewhat skeptical about using them for process inference. This, of course, is not to say that some other, more subtle property would not one day be discovered that carries the signature of the underlying dynamics. There is in fact ongoing work to determine how often it is possible to reject the neutral model in favor of the stochastic niche model described above based on their SADs (Rael et al. unpubl.).

The EN model, perhaps better termed the HN ('hidden niches') model, has already made good progress on clarifying the role, importance, and potential explanations of multimodal SAD patterns. One question still needs to be answered: would versions of the model that make its hidden portions explicit, such as the ones described in the previous section, be able to produce multimodal SADs in a robust way? In the modified model of Fig. 1, the distribution and overlap of species in the additional trait dimensions (for example, the degree to which hosts share their enemies, which appears to be substantial; Novotny et al. 2002) will presumably influence the tendency for multimodality to arise, while in the other modified model in Fig. 2, the number of species at relatively high densities will be lower than in the original model (compare Fig. 2A' and $\left.2 \mathrm{~B}^{\prime}\right)$. A followup question is then whether multimodality observed in nature is highly repeatable in a given system, or occurs some percentage of the time. In the latter case it might be better explained by our stochastic niche model, or more simply by the neutral model (since they only produce multimodality a certain fraction of times) than a modified $\mathrm{HN}$ model that produces multimodality almost all the time. We leave these questions as future problems to be solved.

\section{Methods}

Our starting point is the EN model of Scheffer and van Nes (2006) and Vergnon et al. (2012):

$\frac{\mathrm{d} N_{i}}{\mathrm{~d} t}=r_{i} N_{i}\left(1-\frac{1}{K_{i}} \sum_{j} \alpha_{i j} N_{j}\right)-g \frac{N_{i}^{2}}{N_{i}^{2}+H^{2}}$

where $r_{i}$ is the maximum per capita growth rate of species $i$, $N_{i}$ is its density, $K_{i}$ its carrying capacity, $\alpha_{i j}$ is the competitive effect of species $j$ on species $i$, and $g$ and $H$ are constants. This can also be written as

$\frac{\mathrm{d} N_{i}}{\mathrm{~d} t}=r_{i} N_{i}\left(1-\frac{1}{K_{i}} \sum_{j} A_{i j} N_{j}\right)$

where

$A_{i j}=\alpha_{i j}+\frac{g K_{i} / r_{i}}{N_{i}^{2}+H^{2}} \delta_{i j}$

is the (effective) competition kernel; $\delta_{i j}$ is the Kronecker symbol, equal to 1 if $i=j$ and to 0 otherwise. In the original EN model, $\alpha_{i j}$ has the form

$\alpha_{i j}=\exp \left(-\frac{w_{i j}^{2}}{\sigma^{2}}\right)$

where $w_{i j}$ is the niche distance between species $i$ and $j$ measured along a circular niche axis of unit length.

To generate Fig. 1 the trait space was chosen to have two circular dimensions. The 'distance' between species was interpreted as the Euclidean distance (we have used other norms as well, and they all lead to the same qualitative results). Initially, 200 species were randomly assigned coordinates in this niche space. Then, Eq. 2 was numerically solved with uniform starting abundances, $r_{i}=K_{i}=1$ and 
$A_{i j}=\exp \left(-\frac{w_{i j}^{4}}{0.2^{4}}\right)$

Notice that we used an exponent of 4 instead of the original 2 (Gaussian case) in the expression above. This choice is justified below in the Addendum.

To generate Fig. 2, we simulated Eq. 2 for 200 randomly placed species along a single circular trait axis with a uniform initial condition. The parameters were $r_{i}=K_{i}=1$, and

$A_{i j}=\exp \left(-\frac{w_{i j}^{4}}{0.2^{4}}\right)+0.2 \delta_{i j}$

for Fig. 2A and 2A' and

$A_{i j}=\exp \left(-\frac{w_{i j}^{4}}{0.2^{4}}\right)+0.2 \exp \left(-\frac{w_{i j}^{4}}{0.01^{4}}\right)$

for Fig. 2B and 2B'.

In the stochastic version of the same model, used in making Fig. 3 and 4, the birth, death, and immigration events are modeled as a Poisson process, with their respective rates for species $i$ being

$b_{i}=r N_{i}(1-m)+J m p_{i}$

$d_{i}=\frac{r N_{i}}{K} \sum_{j=1}^{S} \alpha_{i j} N_{j}$

$S_{k}=J m\left(1-\sum_{j=1}^{S} p_{j}\right)$

where $S$ is the number of species present in the local community, $m=0.098$ is the immigration rate, $J=21455$ is the total community size, and $p_{i}$ is the relative abundance of species $i$ in the metacommunity.

The value of $J$ was chosen to be equal to the number of individual trees of diameter at breast height $10 \mathrm{~cm}$ and above on a 50 ha plot on Barro Colorado Island (Hubbell 2001). The parameter $m$ was chosen to be equal to that providing the best fit of the neutral model to the species abundance distribution observed for that same community, which is not far off of what is considered realistic based on measured dispersal kernels (Chisholm and Lichstein 2009).

The distribution of metacommunity abundances $p_{i}$ was chosen following Eq. 34 and 35 of Etienne et al. (2007), i.e. based on the assumption of an infinite neutral metacommunity. The quantity $s_{k}$ is the rate of immigration of species not yet present in the local community; the new species' identity is then determined based on the distribution of metacommunity abundances.

The model was stochastically simulated using the Gillespie algorithm until stationary equilibrium was reached. In the first 100 simulations we set $\alpha_{i j}=\exp \left(-w_{i j}^{4} / \sigma^{4}\right)$ with $\sigma=0.15$ and $K=5400$ (Fig. 4A). In the second 100 simulations we set $\alpha_{i j}=1$ and $K=J$ (neutral dynamics, Fig. 4B). We fitted mixtures of one and two Poisson lognormal distributions to each of our runs following the code ( $\mathrm{R}$ Development Core Team) and methodology described by Dornelas and Connolly (2008), recording the number of cases in which the stationary SAD was significantly multimodal.

\section{Addendum: the form of the competition kernel}

The original EN model uses constant carrying capacities and Gaussian competition coefficients. Though these might seem like reasonable choices, they in fact are a very special combination that should be avoided in studies of species coexistence and limiting similarity. The reason is that, when this combination is properly implemented, it leads to the coexistence of all species, with no competitive exclusion at all (Pigolotti et al. 2010). It can be shown however that an arbitrarily small perturbation of these parameters is enough to take us out of this very special pattern, to one where at equilibrium species are more or less evenly spaced (Gyllenberg and Meszéna 2005, Pigolotti et al. 2010, Barabás et al. 2012). As nature is a noisy place, one should not expect all carrying capacities to be exactly equal, or the competition kernel to be precisely Gaussian - leading one out of this special scenario.

The reason why Scheffer and van Nes (2006) did not observe continuous coexistence in their study was the way they implemented the periodic boundary conditions. In effect, their approach truncates the competition kernel at the ends. This perturbation is enough to destroy the continuous coexistence pattern, but only if the kernel's variance is high enough. For too low variances, since the Gaussian function goes to zero so fast, the truncation effect might not even register numerically on a computer with standard float precision. In effect then, by choosing a constant $K$ and Gaussian kernel, the patterns are dominated by second-order ecological effects, because the system is right on the boundary of stability and instability (Pigolotti et al. 2010, Barabás et al. 2012).

To steer safe of model artifacts stemming from an overly special choice of parameters, one can either perturb the carrying capacities or modify the shape of the competition kernel. Here we opted for the second choice and used kernels of the form $\exp \left(-w_{i j}^{4} / \sigma^{4}\right)$ that are more 'boxy' than the original Gaussian. Pigolotti et al. (2010) show that this parameter combination never leaves the system on the aforementioned stability boundary. The qualitative patterns one obtains with the use of the modified kernel are unaffected; compare our Fig. 1B, 1C and 2A' with Fig. 2B of Scheffer and van Nes (2006) or Fig. 3B of Vergnon et al. (2012).

It is possible however that the particular choice one makes for the exponent of the competition kernel might influence the quantitative specifics of the clustering patterns and quantitative tendency towards multimodality in our stochastic niche model. We have not yet explored this. We note however that there is no clear evidence that the competition kernel takes on any one particular form commonly in nature (Abrams 1975, Meszéna et al. 2006, Hernandez-Garcia et al. 2009, Barabás et al. 2012), so ultimately assessing the tendency of stochastic Lotka-Volterra models to produce multimodality should involve the exploration of a wide range of competition kernels. 
Acknowledgements - We would like to thank Maria Dornelas for her advice on fitting multimodal distributions, and Dávid Völgyes for discussions concerning stochastic processes. We would also like to thank Egbert H. van Nes and Marten Scheffer, and convey our very special thanks to Remi Vergnon, for their willingness to provide information about and help facilitate the discussion of their EN model. This material is based upon work supported by the National Science Foundation under grant no. 1038678, 'Niche versus neutral structure in populations and communities', funded by the Advancing Theory in Biology program. GM acknowledges support by the Hungarian Scientific Research Fund (grant OTKA K81628).

\section{References}

Abrams, P. A. 1975. Limiting similarity and the form of the competition coefficient. - Theor. Popul. Biol. 8: 356-375.

Adler, F. R. and Mosquera, J. 2000. Is space necessary? Interference competition and limits to biodiversity. - Ecology 81: 3226-3232.

Adler, P. B. et al. 2007. A niche for neutrality. - Ecol. Lett. 10: 95-104.

Amarasekare, P. 2003. Competitive coexistence in spatially structured environments: a synthesis. - Ecol. Lett. 6: 1109-1122.

Barabás, G. et al. 2012. Continuous coexistence or discrete species? A new review of an old question. - Evol. Ecol. Res. 14: 523-554.

Barabás, G. et al. 2013. Species packing in nonsmooth competition models. - Theor. Ecol. 6: 1-19.

Bonsall, M. B. et al. 2004. Life history tradeoffs assemble ecological guilds. - Science 306: 111-114.

Chase, J. M. and Leibold, M. A. 2003. Ecological niches: linking classical and contemporary approaches. - Univ. Chicago Press.

Chave, J. et al. 2002. Comparing classical community models: theoretical consequences for patterns of diversity. - Am. Nat. 159: 1-23.

Chesson, P. 1991. A need for niches? - Trends Ecol. Evol. 6: 26-28.

Chesson, P. 2000. Mechanisms of maintenance of species diversity. - Annu. Rev. Ecol. Syst. 31: 343-366.

Chesson, P. et al. 2001. Environmental niches and ecosystem functioning. - In: Kinzig, A. et al. (eds), The functional consequences of biodiversity. Princeton Univ. Press, pp. 213-245.

Chisholm, R. A. and Lichstein, J. W. 2009. Linking dispersal, immigration and scale in the neutral theory of biodiversity. - Ecol. Lett. 12: 1385-1393.

Dornelas, M. and Connolly, S. R. 2008. Multiple modes in a coral species abundance distribution. - Ecol. Lett. 11: 1008-1016.

Etienne, R. S. et al. 2007. The zero-sum assumption in neutral biodiversity theory. - J. Theor. Biol. 248: 522-536.

Gravel, D. et al. 2006. Reconciling niche and neutrality: the continuum hypothesis. - Ecol. Lett. 9: 399-409.

Gyllenberg, M. and Meszéna, G. 2005. On the impossibility of the coexistence of infinitely many strategies. - J. Math. Biol. 50: 133-160.
Haegeman, B. and Loreau, M. 2011. A mathematical synthesis of niche and neutral theories in community ecology. - J. Theor. Biol. 269: 150-165.

Hérault, B. 2007. Reconciling niche and neutrality through the emergent group approach. - Persp. Plant Ecol. 9: 71-78.

Hernandez-Garcia, E. et al. 2009. Species competition: coexistence, exclusion and clustering. - Phil. Trans. R. Soc. A 367: 3183-3195.

Holt, R. D. 2006. Emergent neutrality. - Trends Ecol. Evol. 21: $531-533$.

Hubbell, S. P. 2001. The unified neutral theory of biodiversity and biogeography. - Princeton Univ. Press.

Hubbell, S. P. 2006. Neutral theory and the evolution of ecological equivalence. - Ecology 87: 1387-1398.

Leibold, M. A. 1995. The niche concept revisited: mechanistic models and community context. - Ecology 76: 1371-1382.

Levine, J. M. and HilleRisLambers, J. 2009. The importance of niches for the maintenance of species diversity. - Nature 461: 254-258.

MacArthur, R. H. 1960. On the relative abundance of species. - Am. Nat. 94: 25-36.

MacArthur, R. H. 1970. Species packing and competitive equilibria for many species. - Theor. Popul. Biol. 1: 1-11.

McGill, B. J. 2003. Strong and weak tests of macroecological theory. - Oikos 102: 679-685.

McGill, B. J. et al. 2007. Species abundance distributions: moving beyond single prediction theories to integration within an ecological framework. - Ecol. Lett. 10: 995-1015.

Meszéna, G. 2005. Adaptive dynamics: the continuity argument. - J. Evol. Biol. 18: 1182-1185.

Meszéna, G. et al. 2006. Competitive exclusion and limiting similarity: a unified theory. - Theor. Popul. Biol. 69: 68-87.

Nee, S. and Colegrave, N. 2006. Ecology: paradox of the clumps. - Nature 441: 417-418.

Novotny, V. et al. 2002. Low host specificity of herbivorous insects in a tropical forest. - Nature 416: 841-844.

Pigolotti, S. et al. 2010. How Gaussian competition leads to lumpy or uniform species distribution. - Theor. Ecol. 3: 89-96.

Scheffer, M. and van Nes, E. 2006. Self-organized similarity, the evolutionary emergence of groups of similar species. - Proc. Natl Acad. Sci. USA 103: 6230-6235.

Segura, A. M. et al. 2011. Emergent neutrality drives phytoplankton species coexistence. - Proc. R. Soc. B 278: 2355-2361.

Sugihara, G. 1989. How do species divide resources? - Am. Nat. 113: 458-463.

Tilman, D. 2004. Niche tradeoffs, neutrality, and community structure: a stochastic theory of resource competition, invasion, and community assembly. - Proc. Natl Acad. Sci. USA 7: 661-668.

Vergnon, R. et al. 2012. Emergent neutrality leads to multimodal species abundance distributions. - Nat. Commun. 3: 663.

Volkov, I. et al. 2003. Neutral theory and relative species abundance in ecology. - Nature 424: 1035-1037.

Yan, B. et al. 2012. Trait assembly of woody plants in communities across sub-alpine gradients: identifying the role of limiting similarity. - J. Veg. Sci. 23: 698-708. 